\title{
The Use of Text Recitation as a Teaching Tool in EFL: A Cross-educational Level Investigation in China
}

\author{
$\mathrm{Xia} \mathrm{Yu}$ \\ Southwest University of Political Science and Law, Southwest University, China \\ E-mail: Sheilayu327@hotmail.com
}

Received: 05-03-2017

Published: 01-09-2017
Accepted: 02-05-2017

doi:10.7575/aiac.ijalel.v.6n.5p.191
Advance Access Published: July 2017

URL: http://dx.doi.org/10.7575/aiac.ijalel.v.6n.5p.191

\begin{abstract}
The purpose of this investigation is to address the situation that although general ELT (English Language Teaching) literature does not endorse the practice of text recitation (TR), recent literature cautiously recommends it as a teaching tool with clear learning objectives, especially in countries with Confucian heritage. This paper therefore investigates why TR is commonly practiced in Chinese schools at all educational levels up to tertiary education. In particular, this inquiry considers teachers' view of why TR is practiced and what its effects are. Furthermore, these views across groups at three educational levels are compared and their perceptions are checked against findings of analogous previous research. Analysis of data reveals that the usefulness of the practice of TR to foreign language teaching is generally acknowledged, though, to varying degrees. The study suggests that the contemporary pedagogic practice of TR in China is starting to bear some basic positive features such as differentiating tasks to suit students' ability and choosing texts to cater for students' interest.
\end{abstract}

Keywords: text recitation, ELT, Chinese learner, cross-educational level, perceptions

\section{Introduction}

'Reading aloud for memorisation' is a traditional learning practice in Chinese literacy education and has later been transferred to foreign language education in China. Price (1970, p. 131) predicts that such traditional methods will die slowly. However, rather than a dying practice, 'reading aloud for memorisation' is still widely practiced in schools throughout China (Ding, 2004; see also Rao, 1996; Jin \& Cortazzi, 2002). Students are found to spend hours memorising texts and some teachers require individual students to recite these texts in class or in the teachers' offices (Jin \& Cortazzi, 2002). There are research findings indicating that some successful English learners claim to have intensively employed text recitation (henceforth TR) as a learning strategy (see, e.g., Ding, 2004, 2007; Gao, 2007a; Huang \& Qi, 2005; Wen, 1996). More importantly, College English-Integrated Course (Y.-H. Li, Zhang, \& Wang, 2001), a widely used textbook series for non-English majors in universities, set in each unit a regular assignment of learning by heart several (usually 3 to 4 ) paragraphs in the text. This perhaps serves as an official recognition of the value of the traditional way of text recitation. It seems that Chinese English learners and teachers have not dispensed with this traditional learning method while Economic reforms and the opening of the doors to the West have been in operation for over 30 years and profoundly changed almost every aspect of Chinese society. How can they benefit from the practice with which they might start from kindergarten and continue onwards up even to university?

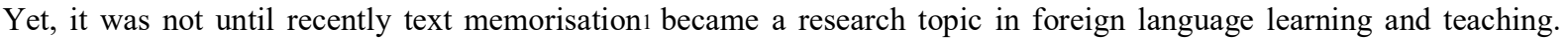
Over the years, TR - in its own right - has been under-investigated especially given the widespread use of the practice in foreign language teaching in some Asian countries as well as China. A gap left by existing studies is that, even if there are reports on learners' beliefs about this practice (e.g., Gao, 2007a; Ting, 2004, 2007; Yu, 2014), there is no formal study of the views of language teachers in regard to TR as a teaching device. The present study, therefore, addresses this gap by examining the practices and beliefs of a group of Chinese teachers regarding the use of TR in foreign language teaching with an attempt to compare the commonalities and diversity across groups from three educational levels which constitute the main part of the English language education system and represent the largest number of English language learners in China.

\section{The study}

\subsection{Research question}

The aim of the current investigation was to seek the purposes and effects of TR in ELT in China. Teachers were given opportunities to express what they had to say about their experiences of TR in their English learning and teaching. The study sets out to answer the following questions: (1) What are Chinese teachers' perceptions of the use of TR in foreign

1 Throughout this paper, the terms text recitation, learning by heart and text memorisation are used more or less with no differentiation. 
language teaching? (2) Are there any commonalities and diversity across groups at different educational levels regarding the teachers' use and beliefs on learning texts by heart?

\subsection{Informants and settings}

The participants in the research include 20 teachers who were teaching English at three different educational levels, i.e. junior high school, senior high school and college. Junior high school is commonly known as Junior Middle School, as from grade 7 to grade 9 for 12-15 years old. Senior high school is commonly known as Senior Middle School as from grade 10 to grade 12 for $15-18$ years old.

All the language teachers participated in a semi-structured interview. 19 of the 20 teachers are English teachers and the remaining one is a Russian teacher with English as her second foreign language. All the university teachers interviewed may be seen as an 'opportunistic sample' (Miles \& Huberman, 1994) in that most of them had been the author's colleagues or schoolmates and the rest the author's acquaintances. The teachers from secondary schools were mostly approached through 'snow-balling', i.e. the teachers interviewed initially introduced their colleagues or friends as potential interview participants.

All the teachers are native Chinese brought up in Confucian Heritage Culture (CHC). While most of them have never been to English speaking countries, four of them had the experience of studying in foreign countries (US, UK and Russia) for one or two years. They are all full-time language teachers working in public schools or private training institutions, and most of them have at least 5 years' teaching experience.

\subsection{Instruments and procedure for data collection}

As Seidman (2006, p. 9) notes that 'at the root of in-depth interviewing is an interest in understanding the lived experience of other people and the meaning they make of that experience'. From this point of view, interviewing serves as an ideal instrument in eliciting the rich and thick data from an 'emic' perspective. In view of the nature of the current research, i.e. to understand the subjective reality of the lived experience of a particular group of individuals, interviews with informants about their lived learning experiences seemed to be the most congruent research strategy. More importantly, given the apparent cultural bearing on the topic under investigation, interviewing 'seems to be the most effective way of bringing the insider's perspective to the analysis, taking us nearer to a description of cultural practices in terms of its members' understandings' (Hyland, 2000, p. 144).

The main method used in eliciting teacher data is the in-depth interview. The interviews are conducted either through face-to-face communication or by telephone. The language used in the interviews is Standard Chinese (Putonghua) or the local dialect spoken in the city where the field work is carried out. The time length ranged from 30 minutes to an hour or so, with one participant being interviewed twice. All the interviews are recorded with high quality recorders and transcribed straightaway.

\section{Results and discussion}

This section reports findings of the interview-based inquiry conducted with selected members of the target group from three educational levels. The subsections are organised according to significant themes which were either informed by the interview questions or emerged from the analysis of the data with respect to the participants' perceptions of their own experiences of using TR in English teaching. In the citations the author makes of the interview data in this section, the coding begins with the participant's name (anonymous). The pseudonym is followed by the identity of the participant, i.e. a teacher-participant $(T P)$ or learner-participant $(L P)_{2}$ and the educational level s/he was in $(J H$ for Junior High, $S H$ for Senior High and $U$ for university).

\subsection{Attitudes towards the use of TR in foreign language teaching}

The teachers' overall attitudes towards the use of TR in their English teaching are reflected in Table 1. More detailed categorisation of the teachers' attitudes by educational level can be also be found in this table.

Table 1. Teachers' Attitudes towards the Use of TR in Foreign Language Teaching $(\mathrm{N}=20)$

\begin{tabular}{lccc}
\hline \hline \multirow{2}{*}{ Attitude } & \multicolumn{3}{c}{ Number of learners expressing attitude } \\
\cline { 2 - 4 } & Junior High(7) & Senior High(5) & College( 8$)$ \\
\hline Very useful & $5(71 \%)$ & $3(60 \%)$ & $4(50 \%)$ \\
Moderately useful & $2(29 \%)$ & $1(20 \%)$ & $3(38 \%)$ \\
Not sure & $0(0 \%)$ & $0(0 \%)$ & $1(12 \%)$ \\
Not too useful & $0(0 \%)$ & $1(20 \%)$ & $0(0 \%)$ \\
\hline
\end{tabular}

The numbers show that the great majority of teachers positively view TR and back the use of it in foreign language learning. However, there were two teachers who hesitated to give this practice a positive rating. One was teaching in senior high school who talked about the issue rather pragmatically:

2 The current study is part of a larger research project with learners' participation. 
I don't require my students to recite texts, but ask them to memorise useful phrases and sentences. The texts in senior high school are usually long so that it is not very practical [to memorise texts]. And the key issue is: What's the purpose of learning texts by heart? It doesn't have much to do with exams. (Wangting, TP, SH)

The other nonconformist is a university teacher who repeatedly used the expression 'it depends' (Wangshu, TP, U) by which she actually emphasised that materials chosen for memorisation really matter:

To let the students memorise texts in the textbook is not very meaningful - they forget next week if they memorise this week. ... They should learn by heart some classic stuff written by masters - better those that are concise in words and profound in meaning so that they can quote it somewhere in their own writing if appropriate. (Wangshu, TP, U)

Interestingly, although this participant was not sure whether TR is a very useful practice in foreign language teaching and learning, she did say the following at the beginning of the interview: 'TR as a way of learning is advantageous and has something to do with [successful] foreign language learning' (Wangshu, TP, U).

While the overall attitudes of teacher participants from the three educational levels were very similar, the teachers in junior high schools seem to be most enthusiastic about the use of TR in that all of them rate it positively and over $70 \%$ of them perceive it to be 'very useful' (see Table 1). This is consistent with an almost unanimous perception emerging from the interview data:

$\ldots$ it is absolutely necessary for students to do this [TR] at the beginning stage. (Jiajie, TP, JH)

... recitation is something essential in foreign language learning, especially for beginners. (Shuqiong, TP, U)

\subsection{Reasons for the use of TR in foreign language teaching in China}

Teachers justified their positive rating of the use of TR in foreign language teaching with a number of strongly argued points. Although their justifications were mainly from a subjective perspective, they also mentioned a few objective reasons why the practice has been in existence in foreign language teaching in China. These context-constrained reasons have been listed in Table 2 with the number of participants referring to them from each teacher group. The quantitative tally, however, needs to be treated with caution. These reasons were all mentioned in passing by the participants when they were responding to a set of predetermined questions or the interviewers' spontaneous follow-up questions. The fact that some teachers did not mention a particular reason does not necessarily mean that they did not share it, but it may indicate it is not of great significance or concern to them.

Table 2. Teachers' Reasons for the Use of TR in Foreign Language Teaching $(\mathrm{N}=20)$

\begin{tabular}{lccc}
\hline \hline Reason & \multicolumn{3}{c}{ Number of learners mentioning reasons } \\
\cline { 2 - 4 } & Junior High(7) & Senior High(5) & College(8) \\
\hline No language environment & 7 & 4 & 7 \\
Test-oriented education & 5 & 2 & 0 \\
Culture of learning & 2 & 1 & 1 \\
\hline
\end{tabular}

All of the teachers supporting the use of TR in foreign language teaching defended their view with reference to the lack of a natural second language environment in China:

For most Chinese, we don't have a language environment so that we have to learn through imitating others. (Yangke, $\mathrm{TP}, \mathrm{SH})$

After all, we don't have natural language input. (Shuqiong, TP, U)

Unlike in foreign countries, we don't have that good condition [in terms of language environment]. (Luyi, TP, U)

These teachers seem to be suggesting that TR is one of the best practices they can choose to cope with an adverse language learning context where 'the limited exposure to English beyond the classroom is a structural condition for every learner and teacher to endure and overcome' (Gao, 2007b, p. 261). As one teacher summarized:

It's all down to the different language environment. ... Although our foreign teacher never thinks it is necessary for students to memorise texts, I still believe it is indispensable in our Chinese condition. (Liangying, TP, JH)

Interestingly, while not having a natural second language acquisition environment is a universal justification among the participants for the existence of TR in China, only four teachers related the practice of TR to traditional Chinese literacy education. One said, 
It is similar to our traditional way of Chinese learning. In addition to extensive reading, some classic texts are required to be recited. After all, both are about learning a language. (Liangying, TP, JH)

Although TR as a way of learning Chinese was rarely used by the participants to defend their use of it in learning English, their belief in the importance of TR might be influenced by their previous experience of learning Chinese. As one student participant mentioned in passing: 'We even do this [TR] when learning our mother tongue. We were required to memorise some texts in Chinese textbooks' (Shuhan, LP, SH). Previous research also documented a learner's relation of mother tongue (Chinese) learning with English learning: 'It is important for a language learner to memorise when learning his or her mother tongue. It is also important for me to memorise certain English texts' (Zhixuan, quoted in Gao, 2007a, p. 213).

Another teacher referred to a 'culture of learning' (Cortazzi and Jin, 1996):

This [memorisation] is a traditional way of learning which is more likely to be acceptable to Chinese. When we start learning something, we tend to commit them to memory. It becomes a mode of entry into learning, which is not necessarily bad. ...There is an issue of learning habit. There is also something to do with Chinese culture of learning. (Yeli, TP, SH)

The 'culture of learning' is undoubtedly a tacit parameter of many of the attitudes or beliefs held by the teachers insomuch as 'any particular culture of learning will have its roots in the educational, and, more broadly, cultural traditions of the community or society in which it is located' (Cortazzi \& Jin, 1996, p. 169). However, the practice of TR is rarely talked about with the awareness of cultural specificity. The 'take-for-granted' concept is evident in a few teachers' utterance: 'I don't believe the foreigners never memorise something when they are learning a language' (Hongying, TP, $U$ ). It seems that cultural discourse has been easily obliterated by awareness of context constraints such as the acquisition-poor foreign language environment and exam-oriented learning. While acknowledging the indelible cultural mark left on many of the comments made by the participants, it is argued here that they hold positive beliefs about TR not necessarily because they consider it to be consistent with traditional Chinese culture and values, as was indicated in some previous research (cf. X.-P. Li, 2005), but more likely because they thought 'there is rationality for its existence in a Chinese context' (Yeli, TP, SH). As pointed by Cheng (2000, p. 101), Chinese culture of learning has become a convenient explanation for any observed learning behaviour of Chinese learners, but Chinese learning strategies are likely to be more complex than they are usually made out to be. Perhaps learners' immediate learning context serves as a better reference point than 'large culture' for understanding Chinese learners.

The most striking difference between teachers from the three educational levels in terms of the context reasons for the use of TR in foreign language teaching was the mention of test-oriented education that China is famous for. It is not surprising that this topic was initiated by most secondary school teachers interviewed, but not by the teachers in college, since secondary schools are under the great pressure from Zhongkao3 and Gaokao4, two high-stakes examinations in China.

What was surprising was the inconsistency among the teachers' comments on TR in relation to test-oriented education. Some secondary teachers, especially senior high school teachers viewed the use of TR as partially being a result of testoriented education. For example:

Learning texts by heart is for their [the students'] better command of grammar and therefore has more to do with exams. We do TR mainly - probably $70 \%$ in degree - for coping with Gaokao. (Yangke, TP, SH)

However, one teacher - the only one who claimed to have not used TR in teaching among all secondary school teachers interviewed - stated that she omitted the practice because she thought it could not effect immediate and obvious benefit in terms of improving the students' performance in exams:

Reading comprehension and cloze tests [in the English exam] test the integrated ability rather than the stuff in the texts. ... Although learning some texts by heart is better than not, the chance of considerably raising the scores in exams [through memorising texts] is very slim. (Wangting, TP, SH)

While the scale was balanced on the issue of whether the use of TR was an indication of the backwash effect of highstakes examinations, the teachers almost unanimously acknowledged the intrinsic value of the practice on top of its utilitarian value:

Of course, during the course of coping with Gaokao [through memorising texts], you naturally improve your oracy and other aspects [of language ability]. (Yangke, TP, SH)

The opinion was further illustrated by one participant as follows:

3 Zhongkao is an informal abbreviation for Senior High School Entrance Examination and Gaokao for College Entrance Examination. 
I think it $[\mathrm{TR}]$ is indispensable for students either in test-oriented education or use-oriented education. ... As for the use-oriented education in which use and speaking is emphasised, it is also beneficial to the students. ... I think we should use more TR in such circumstances. Let me give you an example. We usually have parallel classes and advanced classes in China. You can only have communicative activities successfully carried out in advanced classes, but never in parallel classes. This is because the students in advanced classes have accumulated more and memorised more. (Liangqing, TP, $\mathrm{SH}$ )

In summary, the 'Junior High' group and 'Senior High' group, comparatively, had more correspondence with each other than with the 'College' group in that they both constantly related the practice of TR to exams. Indeed, Chinese learners' emphasis on memorisation is likely to be influenced by the perceived need to gain good scores in examinations (Rao, 2006).

\subsection{Negatives in using TR in foreign language teaching and teachers' countermeasures}

Overall, the teacher participants held an overwhelmingly positive attitude towards the use of TR in foreign language teaching. However, it does not mean that they did not experience difficulties or challenges in applying this practice in practical teaching. Three salient challenges reported by teachers are listed in Table 3 below. From the reading of the table, we can see that all listed challenges were mentioned by secondary teachers. The reason why college teachers did not report any challenges concerning the use of TR as a teaching device may well be due to the fact that, as mentioned early in the paper, memorisation of texts is usually mandatory in secondary schools (especially in junior high schools), but normally operates on voluntary basis at tertiary level.

Table 3. Teachers' Challenges in Using TR in Foreign Language Teaching $(\mathrm{N}=20)$

\begin{tabular}{lccccc}
\hline \hline Problem & \multicolumn{5}{c}{ Number of learners mentioning challenges } \\
\cline { 2 - 6 } & Junior High(7) & Senior High(5) & College(8) \\
\hline Limited time & 3 & 4 & 0 & \\
Keep students' interest & 5 & & 1 & & 0 \\
Differentiation & 6 & 3 & 0 & \\
\hline
\end{tabular}

It needs to be pointed out that although this section concerns teachers' perceived challenges in using TR in their foreign language teaching, some of the teachers' positive pedagogic decisions and practices are also presented in tackling mentioned challenges, which emerged as an interesting theme from the analysis of the interview data.

The most frequently mentioned problem by secondary teachers was the lack of time:

... because time does not allow us to do this [memorising all texts]. (Tangming, TP, JH)

The students have limited time as they have loads of assignments in other subjects. (Jiajie, TP, JH)

In fact, they [students] have very limited time to do TR. (Liangqing, TP, SH)

In coping with the issue of limited time, the teachers usually chose to reduce the amount of memorisation task:

...we teachers don't require them to memorise the whole text or very long paragraphs.... (Liangqing, TP, SH,)

... we do it [TR] selectively. I suggest that the students memorise more paragraphs and sentences. (Tamgming, TP, $\mathrm{JH})$

Some teachers also used alternatives to TR:

It is completely impractical to require students to memorise long articles.... In this case, I will ask them to retell the text in their own words, but at the same time, using the new structures learned in the text. (Tangming, TP, JH)

The second issue often mentioned by most secondary teachers is that of students' tolerance or interest in doing the practice, especially when it comes to young learners. It was generally acknowledged that 'keeping students' interest is very important' (Jiajie, TP, JH) and the encouragement of more text memorisation should be on the premise that '... it does not pose a psychological burden [to the students]' (Liuxia, TP, JH). The choice of material therefore becomes of vital importance:

The choice of the texts for memorisation is obviously important. They should not be too long and too boring. (Yuli, $\mathrm{TP}, \mathrm{JH})$

... we need to choose materials that make sense to the students or interest them in accordance with their ages. (Liuxia, TP, JH) 
One teacher suggested:

... the students may well be given the right to choose one they like among a short list of articles chosen by the teacher because everyone has different interests. They are more likely to be 'using their heart' if they are memorising stuff they are interested in. They will not be very willinghearted if they are forced to recite an article they don't like at all. (Wenna, TP, JH)

Another teacher used the scheme of time allocation to ease the students' psychological burden:

We can increase the frequency of memorisation but decrease the amount each time. It's similar to having more meals but smaller portions. In this way, the students may feel less bored and more easily gain a sense of achievement. (Liuxia, TP, JH)

It is generally agreed among the teachers that teachers' control over the quality as well as quantity of the material for students to memorise is crucial in maximising the benefits and minimise the side-effects of the practice of TR.

Another issue the teachers need to tackle was differentiation in using TR in foreign language teaching. There are two aspects of dealing with the problem: one is about having different students meet different standards or requirements, the other is concerned with adapting the standard of practicing TR. In the first case, the rationale behind the teachers' measure was straightforward; namely, to accommodate the memorisation assignment to the students' ability:

We require the excellent students to recite the whole text and the average the selected paragraphs. As for the poorest group, they only need to be able to read the text aloud in an acceptable manner. (Liangying, TP, JH)

Reading the text aloud as a substitution for learning by heart, though considered by many teachers as qualitatively different from the latter, might be feasible for those who find the task too demanding. According to a participant, 'being fluent in reading aloud is the first step [of learning by heart]' (Tangming, TP, JH). A similar practice was reported in previous study by a Chinese learner: 'It was difficult to memorise the text but I instead read it aloud at least 30 times.' (Interviewee 26, quoted in Jiang, 2008, p. 131). Reading aloud, a learning behaviour that TR normally involves, is 'still widely used in China at every stage of literacy acquisition' (Cortazzi \& Jin, 2010; Parry, 1998, p. 65). Although general ELT methodology literature does not recommend this practice (see, however, Gibson, 2008 for a different argument), interview data in the present study demonstrate a positive response from participants:

I enjoy reading a text aloud. I feel comfortable when I hear my own voice while I say aloud [texts] for memorisation. (Xuying, LP, U)

Sometimes, I read aloud an English newspaper with varying tones. I feel comfortable in doing so. (Yunpeng, LP, U)

In fact, the best way to memorise [a text] is through reading aloud using your mouth. ... Memorising through silent reading is much less effective than reading aloud. (Hongying, TP, U)

These data also echo the following report by Chinese learners in previous research:

I think that it helps improve my linguistic skills when reading these texts aloud for memorisation. For instance, I could improve my intonation ... I think it is important to recite. Recitation is important when learning a language. (Zhixuan, quoted in Gao, 2007a, p. 101).

As for the second point, the rigid practice of verbatim memorisation as in Chinese literacy education was seen as not absolutely necessary as the teachers are aware of the difficulties the students are experiencing:

I noticed the increasing difficulties the students encounter when the texts become more complex. For example, the students complain that some words of the same meaning appear in a text repeatedly like 'often', 'usually' and 'always'. It is hard for them to accurately recall which one is in which sentence. I became more tolerant in such cases. I allow them to use these words interchangeably. ... We don't need to require the students to recite verbatim without any change which is insignificant. (Jiajie, TP, JH)

As mentioned earlier, the aforementioned challenges (limited time and keeping students' interest and differentiation) were not found in university teachers' comments. Unlike in secondary school, memorisation of texts is no longer a mandatory assignment in college and therefore the teachers have much less control over the students' actual practice. The attitude expressed in the following account is not atypical among college teachers:

To memorise [texts] or not is a matter of their [students'] own choice. They may not like this method, or they don't see the need to use it because they are already good enough. As a teacher, I would suggest them to use this method as I know it should be beneficial to them if they really understand the purpose of the practice and persevere at doing it. (Qinxin, TP, U) 
The fact that university teachers are normally much less involved in the monitoring process of students' memorisation of texts does not mean that this practice is not emphasised institutionally at tertiary level. College students are still expected to memorise a certain amount of texts to improve their linguistic competence, as is demonstrated in the following account:

For foundation-laying modules like Integrated English and Advanced English, which are designed to improve [students'] linguistic competence, there are chosen texts for students to memorise in each unit. The lecturers in charge of these modules co-decided which texts are selected for memorisation. ... One of these texts will be tested in the final term examination. (Jiean, TP, U)

While this teacher is talking about the case of teaching students majoring in English, the practice of TR is also encouraged among non-English majors in some colleges:

In the textbook [College English - Integrated Course (Y.-H. Li, et al., 2001)] we are using, there is a regular assignment of learning by heart several paragraphs in the main text in each unit. In order to urge students to do this, we have a special section called 'cloze test' in the final examination paper. Students are required to fill in the missing words or phrases in one or two paragraphs chosen from those they are expected to memorise. (Xiaohong, TP, U)

\section{Conclusion}

In the previous sections findings and discussions have been presented that address the research questions of the study. Although it is difficult to present a conclusive summary of the participants' views, due to the relatively small size of the sample and the diversity of the participants' background, it is clear that the usefulness of the practice of TR to foreign language teaching is generally acknowledged, though, to varying degrees. It is interesting to note that TR, a timehonoured practice originated from traditional Chinese literacy education, continues to be valued in contemporary Chinese foreign language education. Recent research on learners' perspective (e.g., Ting, 2007; Yu, 2014) has reported that the practice of TR are believed to benefit foreign language learning in terms of cultivation of 'language sense', the facilitation of conscious learning and promotion of self-confidence and a sense of achievement, a viewpoint with which many a participant in the current study showed agreement.

In terms of diversity of the practice of TR in three educational levels of teaching, the practical use of TR are more frequently reported by secondary teachers (especially junior high school teachers) than their university counterparts. Moreover, secondary teachers are found to be immensely involved in the practice whereas the university teachers only serve as an advisor. This may be in line with the institutional practice that TR is in most cases an obligatory assignment in secondary schools, especially in junior high schools, but a voluntary choice at tertiary level.

If the relatively small sample used in this study can be taken as indicative, it would appear that the contemporary pedagogic practice of TR in China is starting to bear some basic positive features such as differentiating tasks to suit students' ability and choosing texts to cater for students' interest. Chinese teachers seem to be carefully studying this traditional practice, making changes echoing what constitutes a humanistic view of learning. It is of course the teachers' professional commitment to explore practical techniques (oral presentation, for instance) so as to minimize the intrinsic side-effects of the practice in foreign language teaching.

\section{Acknowledgements}

This research is Supported by Teaching Reform Project in Chongqing Tertiary Education (153040); Post-funded project of Southwest University of Political Science and Law (2014XZHQ-02).

\section{References}

Cheng, X.-T. (2000). Asian students' reticence revisited. System, 28(3), 435-446.

Cortazzi, M., \& Jin, L.-X. (1996). English teaching and learning in China. Language Teaching, 29(2), 61-80.

Cortazzi, M., \& Jin, L.-X. (2010). Creativity and criticality: developing Chinese approaches to learning. Paper presented at the Transnational Dialogues in HE: Responding to the Needs of the Asian Learner in Higher Education.

Ding, Y.-R. (2004). Learning English Text by Heart in a Chinese University. Xi'an: Shanxi Normal University Press.

Ding, Y.-R. (2007). Text memorization and imitation: The practices of successful Chinese learners of English. System, $35(1), 271-280$.

Gao, X.-S. (2007a). From the Chinese mainland to Hongkong: understanding shifts in mainland and Chinese students' English learning strategy use. Unpublished $\mathrm{PhD}$ thesis, University of Hongkong, Hongkong.

Gao, X.-S. (2007b). A tale of Blue Rain Café: A study on the online narrative construction about a community of English learners on the Chinese mainland. System, 35(2), 259-270.

Huang, Q., \& Qi, Q. (Eds.). (2005). Speaking for Success. Shanghai: Shanghai Foreign Language Education Press.

Hyland, K. (2000). Disciplinary Discourses: Social Interaction in Academic Writing. New York: Longman. 
Jiang, X.-L. (2008). Constructing concepts of learner autonomy in language education in the Chinese context: a narrative-based inquiry into university students' conceptions of successful English Language Learning. Unpublished $\mathrm{PhD}$, Warwick University.

Jin, L.-X., \& Cortazzi, M. (2002). English language teaching in China: A bridge to the future. Asian-Pacific Journal of Education, 22(2), 53-64.

Li, X.-P. (2005). An analysis of Chinese EFL learners' beliefs about the role of rote Learning in vocabulary learning strategies. Asian EFL Journal, 7(4), 109-110.

Li, Y.-H., Zhang, P.-R., \& Wang, H.-X. (Eds.). (2001). College English-Integrated Course, Book 1-4. Shanghai: Shanghai Foreign Language Education Press.

Miles, M. B., \& Huberman, A. M. (1994). Qualitative Data Analysis: An expanded Source Book (2nd edn). Thousand Oaks: Sage.

Parry, K. (Ed.). (1998). Culture, Literacy, and Learning English: Voices from the Chinese Classroom. Portsmouth, NH: Heinemann.

Price, R. F. (1970). Education in Modern China. London, Boston and Henley: Routledge \& Kegan Paul.

Rao, Z.-H. (2006). Understing Chinese students' use of language learning strategies from cultural and educational perspectives. Journal of Multilingual and Multicultural Development, 27(6), 491-508.

Seidman, I. (2006). Interviewing as Qualitative Research. New York and London: Teachers College press, Teachers college, Columbia University.

Ting, Y. (2004). Learning English Text by Heart in a Chinese University. Xi'an: Shanxi Normal University Press.

Ting, Y. (2007). Text memorization and imitation: The practices of successful Chinese learners of English. System, 35(1), 271-280.

Wen, Q.-F. (1996). English Learning Strategies. Shanghai: Shanghai Foreign Language Education Press.

Yu, X. (2014). The Use of Textual Memorisation in Foreign Language Learning: Hearing the Chinese Learner and Teacher Voice. TESOL Journal, 5(4), 654-677. 